\title{
Towards Generation of Alternate Electrical Energy via Paddling Impact: Protracted Design and Implementation
}

\author{
Hussain Saleem* \\ Department of Computer Science \\ University of Karachi \\ Karachi, Pakistan
}

\author{
M. Sadiq Ali Khan \\ Department of Computer Science \\ University of Karachi \\ Karachi, Pakistan
}

\begin{abstract}
Electricity calamity and scarcity is among one of the burning questions of third world countries especially in Pakistan these days. There exist multiple other ways to generate electrical energy. In order to address one of the innovative alternate method of electricity generation, we have worked on the technique to generate electricity i.e. using paddling impact. This is done by applying paddling force on floor which is converted to kinetic energy and then to mechanical energy, thereafter mechanical energy is further transformed into electrical energy which is accumulated for genuine use towards power hungry load. The phenomena works on the Law of Conservation of Energy. Undoubtedly this method cannot meet the total requirement of large number of consumers but this can handle energy crisis to some magnitude which seems tremendous and significant accomplishment. This electricity production is not only pollution free but it is more economical since it require low maintenance efforts and can minimize the ongoing burden of charging batteries for hand carrying electrical appliances and street lights etc. Although some engineers, researchers and companies have initiated to provide such solution but lot of work is needed to design for economical solution to be available to every consumer. In this paper we have presented the extended design and implementation.
\end{abstract}

\section{General Terms}

Alternate Resource, Control, Electricity Economy, Energy Conversion, Paddling Impact.

\section{Keywords}

Electricity Generation, Energy Crisis, Mechatronics, Computation, Software Control.

\section{INTRODUCTION}

This has become vibrant requirement that an alternate, economical, monopoly independent, handy and easy to install solution to overcome the crisis of electrical energy must be determined by scientists and engineers. There is a strong need of electrical power that could become promising as an alternate source with robust technology which may be consumed to empower usability of mandatory needed routine appliances e.g. to power street lights, lighting day time dark corridors usually at underpasses, charging cell phones, laptops, emergency light for underground shopping malls, in the absence of main electric line supply as a handy substitute. We have tried to explore the method that how scientists and engineers are striving hard to bring use of the effect to generate alternate electrical power via paddling on floor tiles.

Study, Experimentation, and Manuscript Development: 2008-2012

Article Received by IJCA: April 2013

Review Completed \& Acceptance: May 2013

Suggested revision submitted to IJCA: November 2014

Published: December 2014

*Corresponding Author Email: hussainsaleem@uok.edu.pk
The implementation discussed in this paper is all about developing mechanics with software control for producing electricity. The method is based on the technique where people in their routine walk, passes over the power generating floor made up of tiles each containing an assembly that absorb force in the form of potential energy that is transformed into electrical energy with electro-mechanical setup with software based control and monitor towards power hungry loads. Walking is undoubtedly the most recurrent action of a person during a single day and in daily routine span. Whenever a person of any age with certain weight walks, he exerts potential force towards the floor by means of padding or jumping. The human body mass is therefore accumulated with potential energy when he stand or walk as shown in Figure-1.

The model relies on utilizing this accumulated energy dropped on floor containing tiles, which comprises of an assembly beneath the tile which converts the potential energy to kinetic energy. The kinetic energy is further converted into mechanical energy using Motor-Generator MG-Set which produces electrical power, ready for storage or consumption. Despite this is a very little contribution towards the shortage of electricity but it is very credible development inaugural to business community and economical solution which can fill the scarcity gap of electrical power. This method of electricity production can be considered clean and pollution free for equally to health and environment friendly. Since, this development does not consume any fuel or adiabatic resource; hence its creation leads to null or zero pollution. Figure-2 depicts this phenomenon.

The Japanese firms, who also are deeply dependent on imported fuel like petroleum, oil, coal, gasoline, and firewood to power its industries, are at the vanguard of research into clean and re-useable energy sources. Trials have been carried out at two of the Japanese capital's busiest railway stations installed with such flooring tiles in front of coupon ticketing barriers. Whenever a passerby steps on the floor carpet, the trigger on floor tile happen with a small vibration that is further stored as energy. According to news report of East

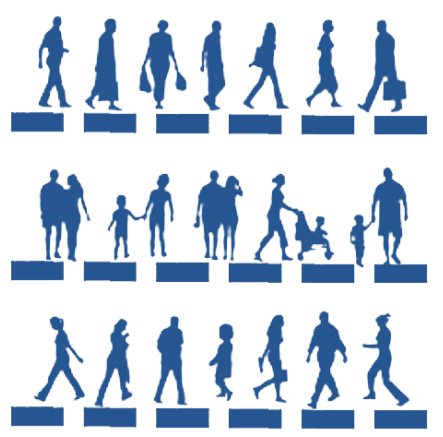

Fig.-1: People exert force while walking on floor 


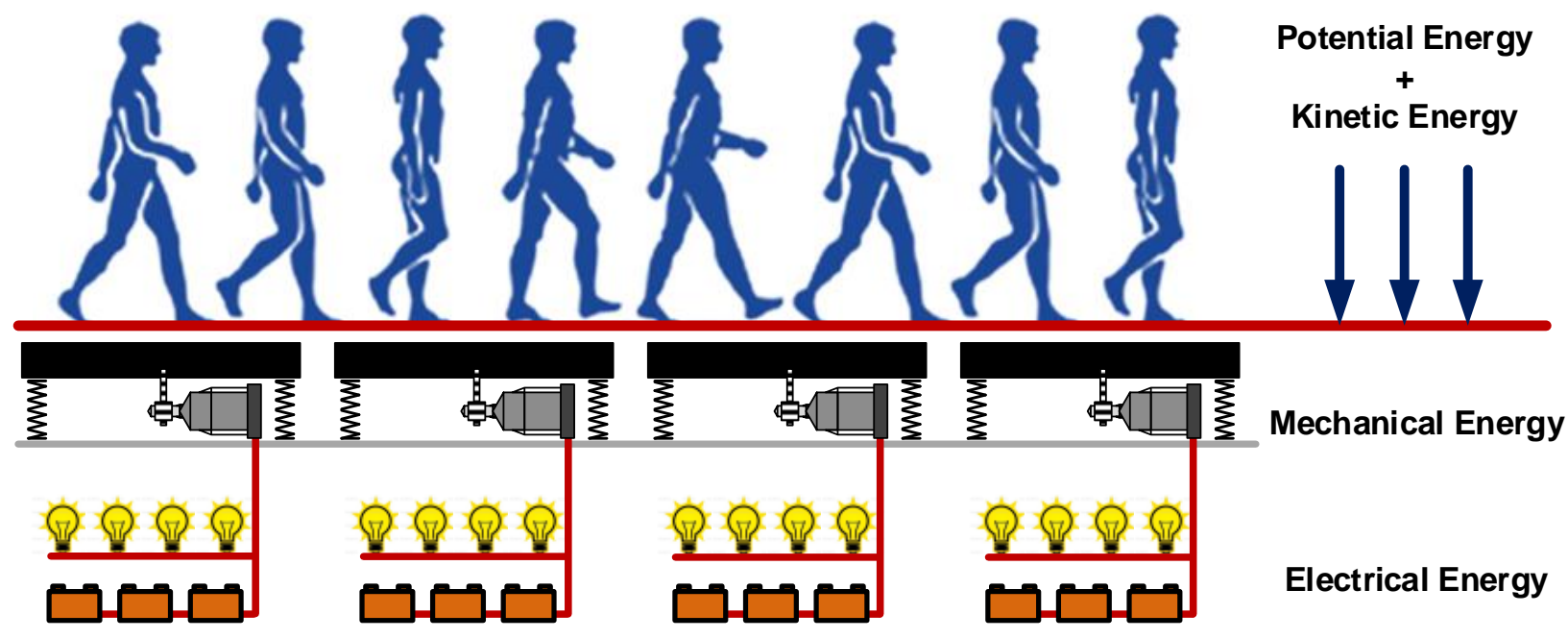

Fig.-2: Implementation of Law of Conservation of Energy

Japan Railway (JR East), the multiplicity resulting approximately 400,000 people who routinely walk through Tokyo Station on average per day generating sufficient energy to light up electronic signboards without need of additional power source. Likewise on an average week day, on the other sideways of Tokyo, a notable 2.4 million people walk through the rambling Shibuya Station where for experimental testing, a power generation floor is installed by Soundpower Corporation to observe the maximum gain. Yoshiaki Takuya of Soundpower Corporation stated that "An average person, weighing $60 \mathrm{Kg}$, can produce only 0.1 Watt per second triggering two steps across the tile. Tremendously, when they are assumed to cover a large area of floor with thousands of pedestrians padding, stepping or jumping, they can produce large total" [15]. The power can be routed to energy-hungry portions with wire or wireless means of transmission [11]. The wireless transmission of electricity also known as WiTriCity is another innovative way of power distribution to energy-hungry loads. Currently methods are under research and development investigations are on the way to adopt WiTriCity type of power distribution.

\section{DISCUSSION}

The whole development is based on two main fields of Science and Engineering: (1) Physics, Law of Conservation of Energy, i.e. the conversion of Potential Energy to Kinetic energy, and then to mechanical and electrical energy, (2) Mechatronics i.e. Mechanics, Computation, Control and Electronics. The system discussed here is developed on the bases of above Scientific and Engineering principles.

Energy exists in various forms e.g. nuclear energy, thermal energy, gravitational energy, elastic energy, rest energy, electric energy, electromagnetic radiation, and momentum etc. These can be characterized in two core classes: (1) Potential energy and (2) Kinetic energy. In physics, potential energy is the energy of an object or a system which is resultant of the position of the body placed on floor space [13]. Detailed studies have been carried out about the branches of potential energy e.g. Statics, Dynamics, Kinetics, and Kinematics etc. As per applicability here, it is accounted only for mass, gravity, and altitude. Mathematically potential energy is presented by Eq.(1) [2].

$$
\boldsymbol{U}=\boldsymbol{m g h}
$$

Where $\boldsymbol{U}$ is the potential energy of the object relative to its stay on the Earth's surface calculated in joules, $\boldsymbol{m}$ is the mass of the object in kilograms, $\boldsymbol{g}$ is the acceleration due to gravity in $\mathrm{m} / \mathrm{s}^{2}$ (meter per second square), and $\boldsymbol{h}$ is the altitude of the object in meters [8].

In physics, the kinetic energy of an object is the energy produced as a result of its motion [9]. It is further defined as the work required to accelerate a body of a certain mass from rest position with specified velocity. The body retains the kinetic energy which is gained during its acceleration unless its velocity is changed. In classical mechanics, the kinetic energy of a point object or a non-rotating rigid body depends on the mass of the body as well as its velocity. The kinetic energy $\boldsymbol{E}_{\boldsymbol{k}}$ is equal to half of the product of mass and the square of the velocity as mentioned in Eq.(2).

$$
E_{k}=\frac{1}{2} m v^{2}
$$

Where $\boldsymbol{m}$ is the mass measured in kilograms and $\boldsymbol{v}$ is the velocity of the body in meters per second whereas the kinetic energy $\boldsymbol{E}_{\boldsymbol{k}}$ is measured in joules.

Potential energy stored in people mass at the time of walking is converted into Kinetic energy. This triggers the MG-Set to develop angular mechanical energy which is converted to electrical energy as motor contains shaft and rotor following electromagnetic field laws to generate electricity with Lenz's Law.

The law of conservation of energy is very crucial law of Physics that states that for an isolated system, the entire sum of energy remains constant throughout time and is conserved over time. It means for an isolated system, energy is localized and that can change its location inside and within the system. For instance potential energy can be transformed into kinetic energy, then in mechanical energy and thereafter in electrical energy but energy can neither be created nor could be destroyed. It can only be transformed in another form of energy [1],[3],[10],[12],[16].

The system presented here is totally following the above scientific and engineering principles. As discussed above, Mechatronics is the field that deals with the scientific methods of mechanics, computation, control and electronics to design and develop any physical system. In general, Mechatronics is a design process that comprises a mixture of mechanical 


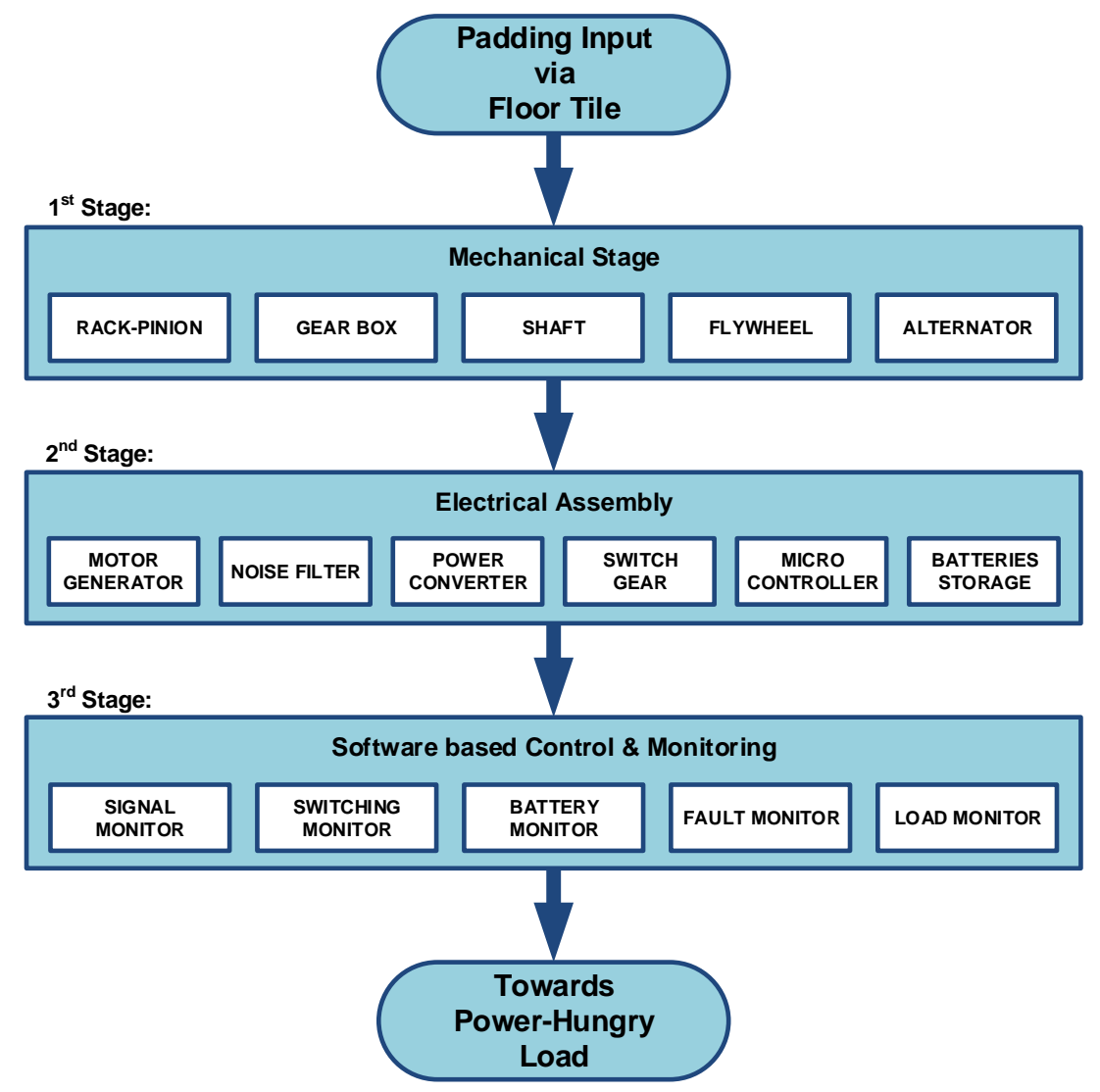

Fig.-3: The Modular Flow diagram of Three Stage Proposed Development Framework

engineering, computer engineering, control engineering, and electronics engineering [14]. A mechatronics engineer combines the design principles and practices of mechanics, electronics, and computing to produce a modest, reasonable and reliable system.

The process followed to generate electricity is carried out here in sequence as when the floor tile is pressed, the pinion in contact with the gear box placed on shaft of the motorgenerator set under the step help the gears to be in motion which is further joined to a flywheel. The flywheel is generating rotations or revolutions per minute (RPM) for the alternator to produce electrical power. Unlike the generator, the alternator needs some power to create power. Increase in torque increases the speed. The generated power is saved in batteries and a control mechanism is developed using microcontroller configuration assembly for voltage regulation and distribution to power hungry loads.

\section{DEVELOPMENT FRAMEWORK}

\subsection{Three Stage Mechatronics Model}

The proposed development framework aims to transform dynamic energy into electrical energy. This framework is composed of Three Stage Functional Mechatronics Model consisting of (1) Mechanical Stage, (2) Electrical Assembly, and (3) Software System for Control and Monitoring. The modular distinctions of the Framework is shown in Figure-3.

While walking on the floor with installed system under carpet, the input in the form of walk is applied on floor tiles i.e. in the form of padding, jumping or pressing tile as shown in Figure-2 with red line, which triggers the Mechanical Stage.

\subsection{Mechanical Stage}

This stage consists of rack-pinion, gear box, shaft, flywheel, and alternator etc. integrated with MG-Set as shown in Figure-4. The rack-pinion is used to convert linear motion to angular momentum. The equilibrium can be expressed by Eq.(3) which is derived from Eq.(2) and Eq.(4). (a)

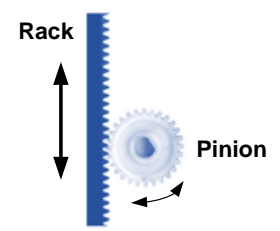

(c)

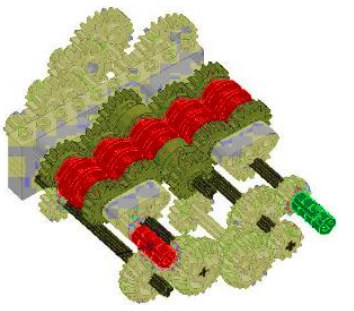

(b)

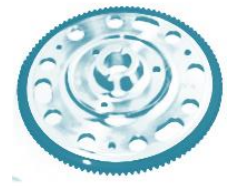

(d)

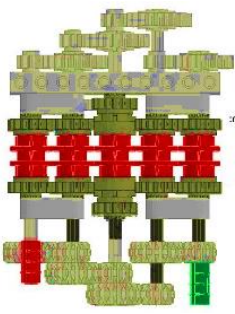

(e)

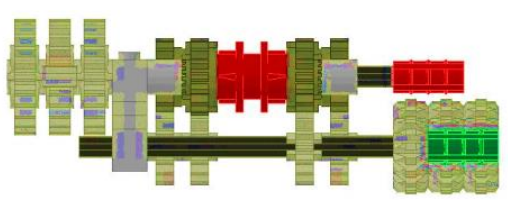

Fig-4: Mechanical components of mechanical stage (a) Rack-Pinion Assembly, (b) Flywheel, (c) Gearbox Assembly, (d) Gearbox - Top view, (e) Gearbox - Side view 


$$
\frac{1}{2} m v^{2}=\frac{1}{2} I \omega^{2}
$$

The pinion is in contact with the shaft which is further connected with gearbox. The gearbox is used to increase or decrease the angular momentum i.e. rotation. A flywheel is a rotating mechanical device which stores rotational energy. It possesses significant moment of inertia due to which it resists changes in rotational speed. The energy accumulated in flywheel is proportional to the square of angular velocity. Additionally, it is a spinning wheel or disc having fixed axle due to which rotation is about one axis only. The Kinetic energy is stored in the rotor as rotational energy. Kinetic energy $\boldsymbol{E}_{\boldsymbol{k}}$ with angular velocity is mathematically expressed by Eq.(4).

$$
E_{k}=\frac{1}{2} I \omega^{2}
$$

Where $\boldsymbol{\omega}$ is the angular velocity, and $\boldsymbol{I}$ is the moment of inertia of the mass about the center of rotation [6]. It is used to stabilize the acceleration of rotor of alternator and produces RPM to drive the rotor of the motor working as generator of electricity.

\subsection{Electrical Assembly}

The electrical assembly consists of various electrical units including motor-generator (MG-Set), noise filter, power converter, switch gear, microcontroller based circuit board, and batteries for storage. The MG-Set converts mechanical power to electrical power which is then stored in batteries and regulated via electronic circuits. MG-Set actually works on the principle of Electrodynamics. The detailed work is required to improve the yield of electricity generation. The vast physics and mathematical concepts of electromagnetic induction where theories of Lorentz force law, Faraday's law, Lenz's law for displacement current, Maxwell's equations, Electromagnetic radiation, Maxwell tensor Poynting vector, Liénard-Wiechert potential, Jefimenko's equations, and Eddy current are required to be monitored for smoothness and efficiency. A noise filter unit is attached to suppress and attenuate the voltage and current jerks, glitches, and fluctuations to produce smooth signal. The power converter unit is included to transform alternating (AC) or direct (DC) signal as per utilization as from (1) AC signal to DC, (2) AC to AC, (3) DC to AC, and (4) DC to DC form. Two methods could be applied to do so: (1) By means of MG-Set, and (2) By means of Power Converter Circuits.

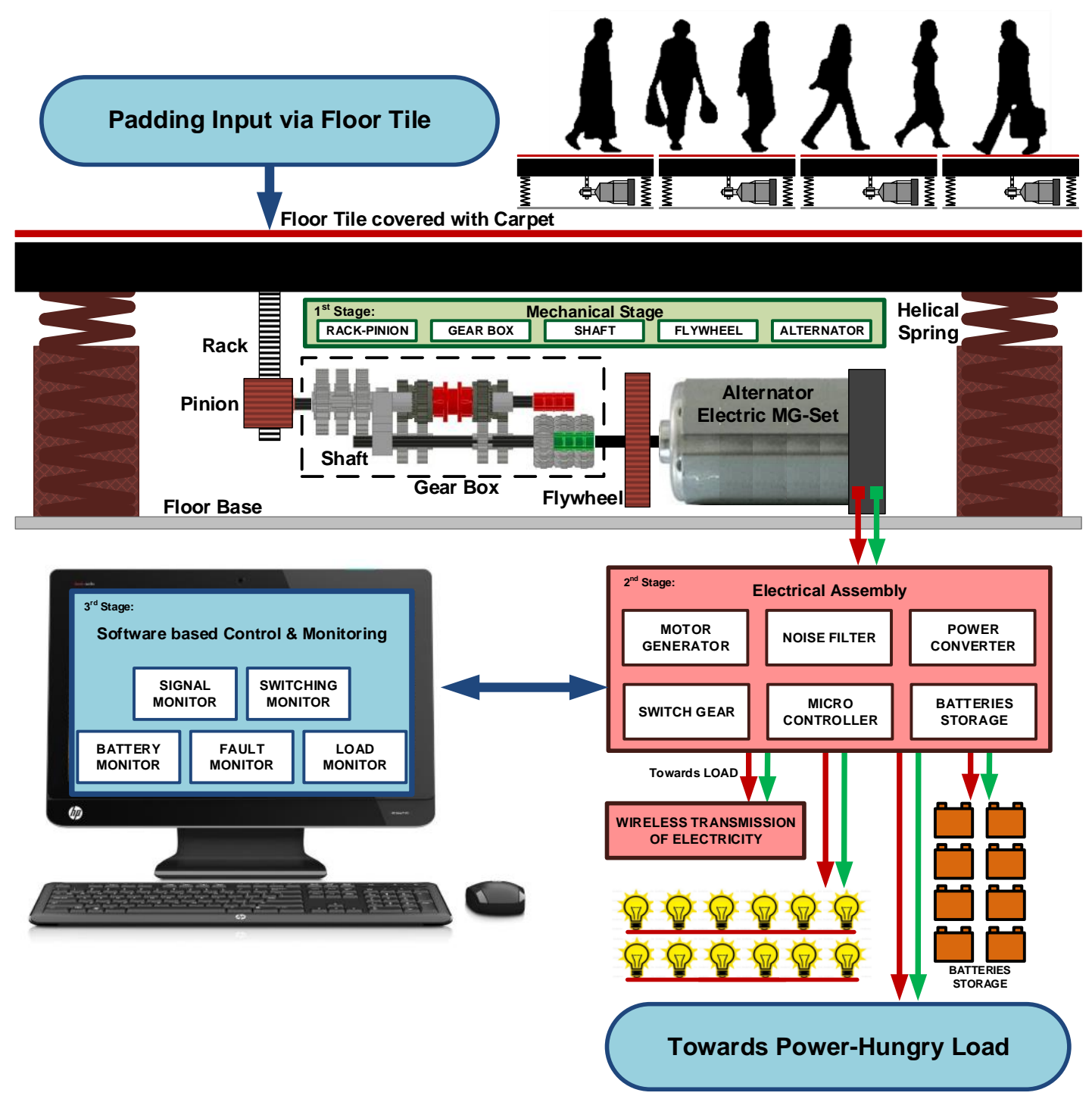

Fig-5: Functional Assembly of the System 
MG-Set can directly be used for various conversions including:

i) $\mathrm{DC}$ at one voltage value to $\mathrm{DC}$ at another voltage value

ii) $\mathrm{DC}$ to $\mathrm{AC}$

iii) $\mathrm{AC}$ at a fixed voltage to $\mathrm{AC}$ of a variable voltage value

iv) $\mathrm{AC}$ at one frequency to $\mathrm{AC}$ at another harmonics frequency

v) $\mathrm{AC}$ single phase to $\mathrm{AC}$ three phase

vi) $\mathrm{AC}$ to $\mathrm{DC}$

Beside MG-Set, the solid state power electronic devices and circuits could be used to develop converter units. These devices includes Diode, SCR (Silicon Controlled Rectifier), Thyristor, GTO (Gate Turn Off Thyristor), Triac, BJT (Bipolar Junction Transistor), Power MOSFET, MCT (MOSControlled Thyristor), IGBT (Insulated Gate Bipolar Transistor), and IGCT (Integrated Gate-Commutated Thyristor).

The power conversion systems can also be used that are classified according to the type of the input and output power requirement.

i) $\mathrm{AC}$ to $\mathrm{DC}$ (Rectifier)

ii) $\mathrm{DC}$ to $\mathrm{AC}$ (Inverter)

iii) $\mathrm{DC}$ to $\mathrm{DC}$ (DC-to-DC Converter)

iv) $\mathrm{AC}$ to $\mathrm{AC}$ (AC-to-AC Transformer)

The optimized and improved above mentioned electronic circuitry of Rectifiers, Inverters, Converters, and Transformers are easily handy available and could be designed on ASITIC Tools [6].

Switch gears are used to switch group and classes of distribution sectors. Optically coupled electronic switches are best for street light application that are available as OEIC (Opto-Electronic Integrated Chip) switch, where Infra-Red IR based Opto-Coupler switching could take place for transmission and receive of switching signal [4],[5]. The RFID technology could also be used for the purpose of switching in nearby vicinity region [7].

An added advantage to our setup is WiTRiCity i.e. "Wireless Transmission of Electricity", a new technology introduced in 2006, provide method of transmit electrical signals by means of resonance. A successful experiment has been carried out at Massachusetts Institute of Technology (MIT) recently to find its usability and limitations [11].

Beside all a Microcontroller chip is placed for programmed distribution to avoid any electricity wastage. This Microcontroller assembly is connected to hardware I/O port to give signal status to Computer Software for controlling the distribution of power with status monitoring.

\subsection{Software based Control \& Monitoring}

This stage is the important control panel that could be placed either nearby or in remotely located control room. The software is developed to control and monitor signal condition, switching, battery strength, fault diagnosis, and load calculation, with replenishment to power hungry devices. The software can monitor large number of floor tile assemblies simultaneously on a certain floor or region. This provides easy to use graphical user interface (GUI). This software also works in network environment and can be accessed and operated remotely by system engineers. All the loads are connected with $3^{\text {rd }}$ Stage assembly and power is furnished according to need determined by software after noise removal, and passing through power converter assembly.

\section{PRINCIPLE OF OPERATION}

In order to understand the principles of operation, we should must understand the functional setup and assembly of the proposed system. A well and preliminary design structure of the system presented in Figure-3 is projected with detailed design in Figure-5, which shows a complete and concrete mechatronics functional assembly of the system consists of three stages. People while walking on the floor exert some potential force on floor surface.

There is a $1^{\text {st }}$ stage called Mechanical stage with integrated mechanical components as explored in Section-3.2. The mechanical assembly is installed under floor tiles that flexibly slide and trigger the rack and pinion. Floor tiles have an elastic support of connected helical springs to the floor base. There is shaft associated with pinion and another shaft associated with MG-Set or Alternator. Both shafts are part of Gear box where multiple gear associations increases the RPM of flywheel. Flywheel is used to stable and maintain rhythm of RPM that operate MG-Set. MG-Set converts mechanical energy to electrical energy.

The Electrical assembly i.e. $2^{\text {nd }}$ stage consists of various sub functional units as discussed in Section-3.3. The electrical signal is conditioned with noise removal is than stored in batteries through some controlled mechanism. This assembly provides connection to WiTRiCity (Wireless Transmission of Electricity) for remote distribution and supply of electrical power. It is further connected to charge the batteries array storage and provide electricity supply to power hungry loads.

The $3^{\text {rd }}$ stage as discussed in Section-3.4 depict a wellconnected Computer System having Software based control and monitoring system equipped with various monitoring capabilities of Electrical sub-units with easy to handle GUI with modules used to monitor signal, switching, batteries, fault and load. The idea of WiTRiCity figures the use of actual procedure about resonance energy that could be created any time persons go walk over the floors [11]. Such power generating floors could be designed with new technological innovations to accumulate and transmit electrical power.

\section{SYSTEM UTILITY}

If such type of mechanism is embedded in places where there is continuous human traffic such as in city malls, railway platforms, city footpaths etc., the electricity generated from these setups can be used for street lights. Proposal for the utilization of waste energy of paddling power with human kinesis is much important for highly populous countries where the crowd is too much observed at roads, bus stands, railway stations, bridges, and shopping malls etc. These are such places where millions of people make crowd and move around the clock. This small scale power production has much credibility as these are easy to be installed.

\section{CONCLUSION}

Third world countries like Pakistan are among those states that are facing electrical energy shortage. This causes disturbance and power breakdown which is main hindrance in economic progress. According to a genuine analysis presented in "The Wall Street Journal" last year, Electricity demand is 25,000 MegaWatts by the end of year 2015, while supply is only 15,000 MegaWatts. There is a deficit of 10,000 MegaWatts that causes power failure and electricity breakdown in power hungry time, creating restlessness among labor in small self-financed industrial setups and among general citizens especially in summer season. We should must adopt this solution to overcome the shortage to some limit. 
Nearly this setup is capable to harvest approximately 100 Watts i.e. 100 Joules per seconds with padding only 12 times on a floor tile. With the scale-up factor of 10 floor tiles with total 120 padding units over same duration, it will produce 1000 Watts. Similarly the installation of 100 floor tiles can produce 1 MegaWatts i.e. 1,000,000 Watts provided simultaneous padding is applied on each floor tile. Approximately 40,000 to 50,000 people routinely walk through a certain corridor per day. We can assume that how much energy could be developed with the multiplicity factor of installation of tiles at busy corridors.

Yoshiaki Takuya of Soundpower Corporation stated that "An average person, weighing $60 \mathrm{Kg}$, can produce only 0.1 Watt per second triggering two steps across the tile. Tremendously, when they are assumed to cover a large area of floor with thousands of pedestrians padding, stepping or jumping, they can produce large total" [15].

\section{REFERENCES}

[1] C. Carathéodory. (1909). "Untersuchungen über die Grundlagen der Thermodynamik". Mathematische Annalen 67: 355-386. doi:10.1007/BF01450409. A mostly reliable translation is to be found at Ref.[10].

[2] Feynman, Richard P. (2011). "Work and potential energy". The Feynman Lectures on Physics, Vol. I. Basic Books. p. 13. ISBN 978-0-465-02493-3.

[3] Giles, R. (1964). "Mathematical Foundations of Thermo dynamics”, Macmillan, New York, pp. 2, 97.

[4] Hussain Saleem et. al. (2012). "A Proposed OEIC Circuit with Two Metal Layer Silicon Waveguide and Low Power Photonic Receiver Circuit", IJCSI International Journal of Computer Science Issues, Vol.9, Issue-5, No.1, September 2012, pp.355-358.

[5] Hussain Saleem et. al. (2012). "A Proposed Silicon Optical Electronic Integrated Circuit with Monolithic Integration of LED, OPFET and Receiver Circuit", IOSR Journal of Computer Engineering (IOSRJCE), Vol.6, Issue-2, (Sep-Oct. 2012), pp.42-46.
[6] Hussain Saleem et. al. (2012). "Review of ASITIC (Analysis and Simulation of Inductors and Transformers for Integrated Circuits) Tool to Design Inductor on Chip", IJCSI International Journal of Computer Science Issues, Vol.9, Issue-4, No.2, July 2012, pp.196-201.

[7] Hussain Saleem et. al. (2012). "Review of Various Aspects of Radio Frequency IDentification (RFID) Technology", IOSR Journal of Computer Engineering (IOSRJCE), Vol-8, Issue-1, (Nov-Dec. 2012), pp.01-06.

[8] Hyperphysics - Gravitational Potential Energy. (Accessed 2013). http://hyperphysics.phy-astr.gsu.edu/Hbase/gpot. html

[9] Jain, Mahesh C. (2009). "Textbook of Engineering Physics - Part-I". PHI Learning Pvt. Ltd. Ch-1, p.9. ISBN 81-203-3862-6.

[10] Kestin, J. (1976). The Second Law of Thermodynamics, Dowden, Hutchinson \& Ross, Stroudsburg PA.

[11] Kotkar, D.; Bhange, A. (2013). "Wireless Transmission of Electricity", Intl. Journal of Scientific \& Engineering Research, Vol.4, No.6, ISSN 2229-5518, pp.142-147.

[12] Landsberg, P.T. (1978). "Thermodynamics and Statistical Mechanics", Oxford University Press, Oxford UK, ISBN 0-19-851142-6, p.79.

[13] McCall, Robert P. (2010). "Energy, Work and Metabolism". Physics of the Human Body. JHU Press. p.74. ISBN 978-0-8018-9455-8.

[14] Mechanical and Mechatronics Engineering Department. "What is Mechatronics Engineering?". Prospective Student Information. University of Waterloo. Retrieved 30 May 2011.

[15] Riyal, Julian. (2008). "Japan harnesses energy from footsteps", http://www.telegraph.co.uk, Accessed. 2013.

[16] Tisza, L. (1966). "Generalized Thermodynamics", MIT Press, Cambridge MA, pp. 50, 109, 123. 